\title{
Nonlinear anomalous diffusion equation and fractal dimension: Exact generalized gaussian solution
}

\author{
I. T. Pedron ${ }^{1}$, R. S. Mendes ${ }^{1}$, L. C. Malacarne ${ }^{1}$, and E. K. Lenzi ${ }^{2}$ \\ ${ }^{1}$ Departamento de Física, Universidade Estadual de Maringá, \\ Avenida Colombo 5790, 87020-900 Maringá-PR, Brazil \\ ${ }^{2}$ Centro Brasileiro de Pesquisas Físicas, R. Dr. Xavier Sigaud 150, 22290-180 Rio de Janeiro-RJ, Brazil
}

(Dated: October 25, 2018)

\begin{abstract}
In this work we incorporate, in a unified way, two anomalous behaviors, the power law and stretched exponential ones, by considering the radial dependence of the $N$-dimensional nonlinear diffusion equation $\partial \rho / \partial t=\nabla \cdot\left(K \nabla \rho^{\nu}\right)-\nabla \cdot(\mu \mathbf{F} \rho)-\alpha \rho$, where $K=D r^{-\theta}, \nu, \theta, \mu$ and $D$ are real parameters and $\alpha$ is a time-dependent source. This equation unifies the O'Shaugnessy-Procaccia anomalous diffusion equation on fractals $(\nu=1)$ and the spherical anomalous diffusion for porous media $(\theta=0)$. An exact spherical symmetric solution of this nonlinear Fokker-Planck equation is obtained, leading to a large class of anomalous behaviors. Stationary solutions for this FokkerPlanck-like equation are also discussed by introducing an effective potential.
\end{abstract}

PACS numbers: 05.20.-y, 05.40.Fb, 05.40.Jc

\section{INTRODUCTION}

Anomalous diffusion has been the subject of numerous investigations in the last years. In particular, anomalous diffusion has played a fundamental role in the analysis of a wide class of systems such as diffusion in plasmas [1], turbulent flows [2], transport of fluid in porous media [3], and diffusion on fractals [4]. In general, anomalous diffusion may be classified employing the mean square displacement, $\left\langle(\Delta x)^{2}\right\rangle$. When $\left\langle(\Delta x)^{2}\right\rangle \propto t^{\eta}$, the value $\eta>1$ characterizes a superdiffusive process, $\eta<1$ a subdiffusive one and $\eta=1$ a 'normal' diffusion.

We consider here a quite large class of anomalous diffusion, namely those associated with the $N$-dimensional nonlinear equation 5 |

$$
\frac{\partial \rho(\mathbf{r}, t)}{\partial t}=\nabla \cdot\left\{K \nabla[\rho(\mathbf{r}, t)]^{\nu}\right\}-\nabla \cdot[\mu \mathbf{F}(\mathbf{r}, t) \rho(\mathbf{r}, t)]-\alpha(t) \rho(\mathbf{r}, t),
$$

where $K=D r^{-\theta}, \nu, \theta$, and $D$ are real parameters, $\mu$ is the drift coefficient, $\mathbf{F}(\mathbf{r}, t)$ is the external force, and $\alpha(t)$ is a time-dependent source. From the Gauss theorem one verifies that this general equation preserves the norm $\int \rho(\mathbf{r}, t) d^{N} r$ if $\alpha(t)=0$ and $K \nabla[\rho(\mathbf{r}, t)]^{\nu}-\mu \mathbf{F}(\mathbf{r}, t) \rho(\mathbf{r}, t)$ goes sufficiently rapid to zero when $r=|\mathbf{x}| \rightarrow \infty$. The difference between the Fokker-Planck-like equation (11) and the usual one is that the diffusion term depends on a power of $\rho(\mathbf{r}, t)$ and the diffusion coefficient is spatially dependent. Notice that this equation for $\theta=0$ and $\alpha(t)=0$ has the form:

$$
\frac{\partial \rho(\mathbf{r}, t)}{\partial t}=D \nabla^{2}[\rho(\mathbf{r}, t)]^{\nu}-\nabla \cdot[\mu \mathbf{F}(\mathbf{r}, t) \rho(\mathbf{r}, t)],
$$

and it is usually called porous media equation. The above equation admits important physical applications such as percolation of gases through porous media [6], thin liquid films spreading under gravity [7], some selforganizing phenomena [8], and surface grow [3], among others (see Ref. [9] and references therein). Furthermore, this equation has been related to nonextensive Tsallis statistics. This connection was first pointed by Plas- tino and Plastino 10 and revisited by others authors [9, 11, 12, 13, 14, 15, 16.

Another important kind of anomalous diffusion emerges from Eq. (11) when $\nu=1, \alpha(t)=0$ and $\mathbf{F}(\mathbf{r}, t)=0$. In this case, the equation

$$
\frac{\partial \rho(\mathbf{r}, t)}{\partial t}=\nabla \cdot[K \nabla \rho(\mathbf{r}, t)]
$$

was related to turbulent diffusion in the atmosphere 17. (see also Ref. 18). Here $K \propto r^{4 / 3}$. In general, in a $d$-dimensional space, $\nabla \cdot K \nabla$ is proportional to $\frac{1}{r^{d-1}} \frac{\partial}{\partial r} r^{d-1-\theta} \frac{\partial}{\partial r}+\frac{A}{r^{2+\theta}}(A$ is an operator, which depends on the angular variables). Thus,

$$
\tilde{\Delta}=\frac{1}{r^{d-1}} \frac{\partial}{\partial r} r^{d-1-\theta} \frac{\partial}{\partial r}
$$

is the radial part of the operator $\nabla \cdot K \nabla$ to be considered in the study of $d$-dimensional spherical symmetrical solutions of Eq. (3). If $d$ is interpreted as a positive real number, it plays a role of a fractal dimension embedding in a $N$-dimensional space. Thus, Eq. (3) becomes, in a fractal 
framework, the O'Shaughnessy-Procaccia equation 19,

$$
\frac{\partial \rho(r, t)}{\partial t}=D \tilde{\Delta} \rho(r, t)
$$

We are ready now to unify Eqs. (2) and (5). In fact, if we consider only diffusive terms and the radial part of these equations we have [20

$$
\frac{\partial \rho(r, t)}{\partial t}=D \tilde{\Delta}[\rho(r, t)]^{\nu}
$$

Of course, if $\theta=0$ and $\nu=1$, the usual diffusion equation is recovered, i.e.,

$$
\frac{\partial \rho(\mathbf{r}, t)}{\partial t}=D \nabla^{2} \rho(\mathbf{r}, t)
$$

In the previous scenario, Eq. (11) can be generalized in order to be also employed in a fractal context, when spherical symmetric solutions are considered and $\nabla \cdot K \nabla$ is replaced by $\tilde{\Delta}$. This paper is dedicated to investigate a class of these solutions in the presence of a source term and with a drift term, as well as the stationary solutions when a general drift term is present.

The present work is organized as follows. In the next section we review an exact solution (generalized gaussian) for Eq. (6) and its limit cases. This generalized gaussian essentially gives the basis for the remaining solutions investigated here. In Sec. III, a formal solution for the nonlinear Fokker-Planck equation Eq. (6) with a source term is obtained. Sec. IV deals with the application of our formal solution for a time power law source. In Sec. V, we present a generalized gaussian solution for the nonlinear Fokker-Planck with a linear spherical force term. Furthermore, in Sec. VI, we consider the stationary solutions for nonlinear Fokker-Planck equation with a general drift term. A summary of our results is presented in the last section.

\section{UNIFIED POWER-LAW AND STRETCHED EXPONENTIAL SOLUTION}

Motivated by the Gaussian solution of the usual diffusion, Eq. (7), it is natural to employ a generalization of such solution when we are investigating Eq. (6). In this direction, we follow Ref. 20 employing the ansatz:

$$
\rho(r, t)=\left[1-(1-q) \beta(t) r^{\lambda}\right]^{1 /(1-q)} / Z(t)
$$

if $1-(1-q) \beta(t) r^{\lambda} \geq 0$, and $\rho(r, t)=0$ if $1-(1-q) \beta(t) r^{\lambda}<$ 0 (cutoff condition). By replacing this generalized gaussian function in Eq.(6), we verify that $\lambda=2+\theta, q=2-\nu$,

$$
\beta(t)=\beta_{0}[1+A t]^{-\lambda /[\lambda+d(1-q)]},
$$

and

$$
Z(t)=Z_{0}[1+A t]^{d /[\lambda+d(1-q)]}
$$

where $A=D \lambda(2-q)[\lambda+d(1-q)] \beta_{0} Z_{0}^{q-1}, \beta_{0}=\beta(0)$ and $Z_{0}=Z(0)$. It is also important to note that the product $\beta Z^{\lambda / d}$ is time independent.

From the solution (8) the mean value for a generic function $f(r)$ can be calculated. In particular, the mean value for $r^{\sigma}$ is given by

$$
\left\langle r^{\sigma}\right\rangle=\frac{\int_{0}^{\infty} r^{\sigma} \rho(r, t) r^{d-1} d r}{\int_{0}^{\infty} \rho(r, t) r^{d-1} d r}=C_{\sigma} \beta^{-\sigma / \lambda}
$$

with

$$
C_{\sigma}=\frac{\Gamma\left(\frac{\sigma+d}{\lambda}\right)}{\Gamma\left(\frac{d}{\lambda}\right)} \begin{cases}\frac{\Gamma\left(\frac{1}{1-q}+\frac{d}{\lambda}+1\right)}{(1-q)^{\sigma / \lambda} \Gamma\left(\frac{\sigma+d}{\lambda}+\frac{1}{1-q}+1\right)} & q<1 \\ \frac{\Gamma\left(\frac{1}{q-1}-\frac{d+\sigma}{\lambda}\right)}{(q-1)^{\sigma / \lambda} \Gamma\left(\frac{1}{q-1}-\frac{d}{\lambda}\right)} & q>1 .\end{cases}
$$

Observe that the existence of $\left\langle r^{\sigma}\right\rangle$ imposes restrictions over the parameters: $\lambda+d(1-q)>\sigma(q-1)$ and $\lambda>0$. In addition, the restriction $q<2(\nu>0)$ is necessary for $\rho(r, t)$ to be real. In the following, we assume that the parameters obey the above restrictions.

An important particular case of $\left\langle r^{\sigma}\right\rangle$ is the mean square displacement, $\left\langle r^{2}\right\rangle$, since such mean value is commonly employed to classify the diffusion process. Thus, by taking $\sigma=2$ in Eqs. (11) and (12) we have

$$
\left\langle r^{2}\right\rangle=C_{2} \beta_{0}^{-2 / \lambda}[1+A t]^{2 /[\lambda+d(1-q)]} .
$$

Consequently, $\left\langle r^{2}\right\rangle \sim t^{2 /[2+\theta+d(\nu-1)]}$ for a sufficient large $t$. Now, it is easy to verify that $\theta=d(1-\nu)$ describes a 'normal' diffusion, even when $\theta \neq 0$ and $\nu \neq 1$. In fact, there is a competition between $\theta$ and $\nu$ in such way that the anomalous diffusive regime induced by $\theta \neq 0$ is compensated by a convenient one with $\nu \neq 1$. Furthermore, this competition leads to a subdiffusive process when $\theta>d(1-\nu)$, and to a superdiffusive one when $\theta<d(1-\nu)$.

Note, also, that an alternative point of view to verify how much a solution deviates from the usual one is to analyze the decay of $\rho(0, t)$. In other words, the way the solution decreases in this central point can inform about its deviation from the normal diffusion. In our case, Eq. (8) gives $\rho(0, t) \sim t^{-d /[2+\theta+d(\nu-1)]}$ in contrast with $\rho(0, t) \sim t^{-d / 2}$, obtained from the usual diffusion.

We remark that important limiting cases can be obtained from Eq. (8). If $\theta=0$, the solution for the porous media equation without the drift term is recovered [9, 10,

$$
\rho(r, t)=\left[1-(1-q) \beta_{1}(t) r^{2}\right]^{\frac{1}{1-q}} / Z_{1}(t)
$$

with $\beta_{1}(t) \sim t^{-2 /[2+d(1-q)]}$ and $Z_{1}(t) \sim t^{d /[2+d(1-q)]}$. On the other hand, for $\nu=1$, the point-source solution for the O'Shaugnessy-Procaccia equation 19] emerges,

$$
\rho(r, t)=e^{-\beta_{2}(t) r^{\theta+2}} / Z_{2}(t)
$$

where $\beta_{2} \sim t^{-1}$ and $Z_{2}(t) \sim t^{d /(2+\theta)}$. Moreover, Eq. (8) recovers the time-translated Gaussian solution for the Eq. 
(7)

$$
\rho(r, t)=\frac{\rho_{0}}{\left[4 \pi D\left(t_{0}+t\right)\right]^{d / 2}} e^{-\frac{r^{2}}{4 D\left(t_{0}+t\right)}} .
$$

In fact, Eqs. (9) and (10) can be respectively written as

$$
\beta(t)=\tilde{A}\left(t_{0}+t\right)^{-\lambda /[\lambda+d(1-q)]}
$$

and

$$
Z(t)=\tilde{B}\left(t_{0}+t\right)^{d /[\lambda+d(1-q)]},
$$

with $\tilde{A}=\beta_{0} A^{-\lambda /[\lambda+d(1-q)]}, \tilde{B}=Z_{0} A^{d /[\lambda+d(1-q)]}$, and $t_{0}=1 / A$. Eq. (16) is obtained from Eq. (8) with $\beta(t)$ and $Z(t)$ given above in the limit $\nu=1$ and $\lambda=2$, and by using the normalization $\Omega_{d} \int \rho(r, t) r^{d-1} d r=\rho_{0}$, where $\Omega_{d}=2 \pi^{d / 2} / \Gamma(d / 2)$ is the solid angle in a $d$-dimensional space.

To conclude this review, we notice that our point-like solution can be connected with the Tsallis entropy 20 .

\section{NONLINEAR FOKKER-PLANCK EQUATION WITH TIME-DEPENDENT SOURCE TERM}

Nonlinear diffusion with absorption arises in many areas of science such as engineering, biophysics, solid state physics and reactor, among others 21, 22, 23, 24. This section is dedicated to the analysis of the generalized Fokker-Planck equation presented in the introduction with a source term. This kind of equation is expected to provide a basis for phenomenological applications, where a large class of nonlinear anomalous diffusion with source plays an important role.

Let us consider the nonlinear Fokker-Planck equation (11). The source term in this equation can be removed by an appropriate change in the solution:

$$
\rho(\mathbf{r}, t)=\exp \left(-\int_{0}^{t} \alpha\left(t^{\prime}\right) d t^{\prime}\right) \hat{\rho}(\mathbf{r}, t) .
$$

In this way, $\hat{\rho}(\mathbf{r}, t)$ obeys the equation

$$
\frac{\partial \hat{\rho}(\mathbf{r}, t)}{\partial t}=\nabla \cdot\left\{\hat{K} \nabla[\hat{\rho}(\mathbf{r}, t)]^{\nu}\right\}-\nabla \cdot[\mu \mathbf{F}(\mathbf{r}, t) \hat{\rho}(\mathbf{r}, t)],
$$

where $\hat{K}(t)=K \exp \left[(1-\nu) \int_{0}^{t} \alpha\left(t^{\prime}\right) d t^{\prime}\right]$. Thus, Eq. (20) has the same form of the corresponding one without the source term, but with a time-dependent diffusion coefficient $\hat{K}$. Note that this time-dependence is induced by the nonlinear term $\rho^{\nu}$, disappearing when $\nu=1$.

As emphasized in the introduction, below Eq. (7), the replacement of the operator $\nabla \cdot(\hat{K} \nabla)$ by $\tilde{\Delta}$ unable us also to analyze situations with fractal dimension in the sense of O'Shaughnessy-Procaccia. Thus, to investigate a radial nonlinear anomalous diffusion equation with a source term and non-integer dimension $d$, we consider

$$
\frac{\partial \rho(r, t)}{\partial t}=D \tilde{\Delta}[\rho(r, t)]^{\nu}-\alpha(t) \rho(r, t)
$$

instead of Eq. (1). In the interests of brevity we omitted the drift term. The case with an external force is discussed in Sec. V. To eliminate the source term, we proceed analogously as in the previous paragraph. Therefore, we obtain

$$
\frac{\partial \hat{\rho}(r, t)}{\partial t}=\hat{D} \tilde{\Delta}[\hat{\rho}(r, t)]^{\nu}
$$

with $\hat{D}(t)=D \exp \left[(1-\nu) \int_{0}^{t} \alpha\left(t^{\prime}\right) d t^{\prime}\right]$. Now, we redefine the time variable introducing an effective time as

$$
\tau(t)=\int_{0}^{t} \hat{D}\left(t^{\prime}\right) d t^{\prime}
$$

so Eq. (22) can be written as

$$
\frac{\partial \hat{\rho}(r, \tau)}{\partial \tau}=\tilde{\Delta}[\hat{\rho}(r, \tau)]^{\nu}
$$

The solution of this equation was presented and discussed in Sec. II. Thus, it is given by Eqs. (8), (9) and (10) with $D=1$ and $t$ replaced by $\tau$.

Another possible generalized Fokker-Planck is

$$
\frac{\partial[\rho(\mathbf{r}, t)]^{\eta}}{\partial t}=\nabla \cdot\left\{K \nabla[\rho(\mathbf{r}, t)]^{\nu}\right\}-\nabla \cdot\left\{\mu \mathbf{F}(\mathbf{r}, t)[\rho(\mathbf{r}, t)]^{\eta}\right\}-\alpha(t)[\rho(\mathbf{r}, t)]^{\eta}
$$


to Eq. (11) when we replace $[\rho(r, t)]^{\eta}$ by $\tilde{\rho}(r, t)$. In the same direction, this kind of generalization can be incorporated in Eq. (21). In this paper, we will not present details about these possibilities because their solutions can be directly obtained from Eqs. (11) or (21) by using the identification $\tilde{\rho}=\rho^{\eta}$.

\section{SOME KINDS OF SOURCES}

In order to have an enhanced understanding of the relevance of the source terms in the anomalous diffusion equation, we apply the previous general procedure to study some specific cases. In this context, the simplest situation to be investigated is when $\alpha(t)=\alpha_{0}$, where $\alpha_{0}$ is a constant. We also investigate a more generic source term, $\alpha(t)=\alpha_{n} t^{n}$.

\section{A. Constant source}

Now, taking into account Eq.(21), we analyze the case $\alpha(t)=\alpha_{0}$. Thus, the effective time, Eq. (23), becomes

$$
\tau(t)=D \frac{\exp \left[(1-\nu) \alpha_{0} t\right]-1}{(1-\nu) \alpha_{0}}
$$

This effective time exhibits three different behaviors. For $(1-\nu) \alpha_{0}<0$, the effective time goes exponentially to $\tau_{\infty}=D /\left[(\nu-1) \alpha_{0}\right]$, i.e., a stabilization occurs in the diffusive process. For $(1-\nu) \alpha_{0}>0$, there is an exponential increase leading to $\tau(t) \approx D \exp \left[(1-\nu) \alpha_{0} t\right] /\left[(1-\nu) \alpha_{0}\right]$, when $t$ is large. It is interesting to remind that this kind of behavior arises due to the nonlinearity of the diffusion equation. In this direction, we have $\tau=D t$ when $\nu=1$, which corresponds to $q=1$ as long as $q=2-\nu$.

By using Eqs. (9) and (10) with $t$ replaced by $\tau$, we obtain

$$
\beta(\tau(t))=\beta_{0}\left(1+A \frac{\exp \left[(1-\nu) \alpha_{0} t\right]-1}{(1-\nu) \alpha_{0}}\right)^{-\lambda /[\lambda+d(1-q)]}
$$

and

$$
Z(\tau(t))=Z_{0}\left(1+A \frac{\exp \left[(1-\nu) \alpha_{0} t\right]-1}{(1-\nu) \alpha_{0}}\right)^{d /[\lambda+d(1-q)]}
$$

Thus, from Eqs.(8) and (19) we verify that

$$
\rho(r, t)=e^{-\alpha_{0} t}\left[1-(1-q) \beta(\tau(t)) r^{\lambda}\right]^{1 /(1-q)} / Z(\tau(t))
$$

if $1-(1-q) \beta(\tau(t)) r^{\lambda} \geq 0$, and $\rho(r, t)=0$ if $1-(1-$ q) $\beta(\tau(t)) r^{\lambda}<0$.
In this solution, when $(1-\nu) \alpha_{0}<0$, we have, for large time,

$$
\rho(r, t) \approx e^{-\alpha_{0} t}\left[1-(1-q) \beta_{\infty} r^{\lambda}\right]^{1 /(1-q)} / Z_{\infty}
$$

where

$$
\beta_{\infty}=\beta_{0}\left\{1+A /\left[(\nu-1) \alpha_{0}\right]\right\}^{-\lambda /[\lambda+d(1-q)]}
$$

and

$$
Z_{\infty}=Z_{0}\left\{1+A /\left[(\nu-1) \alpha_{0}\right]\right\}^{d /[\lambda+d(1-q)]} .
$$

This means that $\rho(r, t)$ decays (grows) exponentially in the time when $\alpha_{0}>0\left(\alpha_{0}<0\right)$, and is spatially limited for $q<1$ as long as $\rho(r, t)=0$ if $r^{\lambda} \geq 1 /\left[(1-q) \beta_{\infty}\right]$. In contrast, the spatial asymptotic power law behavior,

$$
\rho(r, t) \sim r^{\lambda /(1-q)},
$$

arises for sufficiently large $t$ and $(1-\nu) \alpha_{0}>0$. On the other hand, when $\nu=1$, we have

$$
\rho(r, t) \sim t^{-d / \lambda} \exp \left[-\alpha_{0} t-r^{\lambda} /\left(D \lambda^{2} t\right)\right]
$$

for large $t$.

Let us focus attention now on the mean square displacement. By using the effective time in Eq. (13), we obtain

$$
\left\langle r^{2}\right\rangle=C_{2} \beta_{0}^{-2 / \lambda}\left[1+A \frac{e^{(1-\nu) \alpha_{0} t}-1}{(1-\nu) \alpha_{0}}\right]^{2 /[\lambda+d(1-q)]} .
$$

The long time behavior for $\left\langle r^{2}\right\rangle$ leads to an exponential growth when $(1-\nu) \alpha_{0}>0$. For $(1-\nu) \alpha_{0}<0$, this expression converges to an asymptotic value. For $\nu=1$, we have $\left\langle r^{2}\right\rangle \sim t^{2 / \lambda}$, i.e., when $\nu=1$, the mean square displacement is not affected by the constant source term. These results show that the presence of a source term plays a relevant role on the diffusive process when $\nu \neq 1$.

\section{B. Source with a general power term}

Now we analyze the more general case corresponding to the source $\alpha_{n}(t)=\alpha_{n} t^{n}$, where $\alpha_{n}$ and $n$ are constants. The related effective time, $\tau_{n}(t)$, is given by

$$
\tau_{n}(t)=\int_{t_{0}}^{t} D \exp \left[\frac{(1-\nu) \alpha_{n}}{n+1}\left(t^{\prime n+1}-t_{0}^{n+1}\right)\right] d t^{\prime},
$$

where $t_{0}$ is a cutoff only used to avoid divergence when $n \leq-1$ and $(1-\nu) \alpha_{n} /(n+1)>0$. When $n>-1$, we get 


$$
\tau_{n}(t)=D \frac{1}{n+1}\left(-\frac{\alpha_{n}(1-\nu)}{1+n}\right)^{-1 /(1+n)}\left[\Gamma\left(\frac{1}{1+n}\right)-\Gamma\left(\frac{1}{1+n}, \frac{\alpha_{n}(\nu-1) t^{1+n}}{1+n}\right)\right] .
$$

Here $\Gamma(c)$ and $\Gamma(c, x)$ are the gamma and the incomplete gamma functions, respectively. By using the asymptotic behavior for $\Gamma(c, x)$, we obtain $\tau_{n} \sim t^{-n} \exp \left[-\alpha_{n}(\nu-\right.$ 1) $\left.t^{1+n} /(1+n)\right]$ for $n>-1$ and large $t$. For $n=-1$, we have $\tau_{-1}(t) \sim t^{\alpha_{-1}(1-\nu)+1}$, and for $n<-1$, we verify that $\tau_{n}(t) \sim t$. Taking into account the asymptotic behavior of the mean square displacement, $\left\langle r^{2}\right\rangle \sim \tau_{n}^{2 /[\lambda+d(1-q)]}$, we identify two different behaviors when $n>-1$ : if $\alpha_{n}(\nu-1)>0$, the mean square displacement reaches a limit value, and it grows exponentially if $\alpha_{n}(\nu-1)<0$. For $n=-1$, its value goes with $t^{2\left[1-(1-q) \alpha_{-1}\right] /[\lambda+d(1-q)]}$. Finally, for $n<-1$, we obtain $\left\langle r^{2}\right\rangle \sim t^{2 /[\lambda+d(1-q)]}$.

\section{EXTERNAL FORCES}

Now we draw our attention in order to analyze the generalized radial Fokker-Planck equation with a drift term. We take Eq. (6) with a radial external force, i.e.,

$$
\frac{\partial \rho(r, t)}{\partial t}=D \tilde{\Delta}[\rho(r, t)]^{\nu}-\frac{1}{r^{d-1}} \frac{\partial}{\partial r}\left[r^{d-1} F(r) \rho(r, t)\right],
$$

where we set $\mu$ equal to unity. In this section we are going to consider a specific case of a particle moving in a harmonic spherical potential $V(r)=k r^{2} / 2$. Thus, $r=0$ is a stable equilibrium position for positive $k$, and $r=0$ is an unstable equilibrium position for negative $k$. Eq. (38), with this potential, corresponds to the UhlenbeckOrnstein process [26] in the particular case of $\theta=0$ and $q=1(\nu=2-q)$.

By using, again, the ansatz (8) into Eq. (38), we verify that $Z(t)$ and $\beta(t)$ obey the equations:

$$
\frac{1}{Z} \frac{d Z}{d t}=D d \nu \lambda \beta Z^{q-1}-k d
$$

and

$$
\frac{1}{\beta} \frac{d \beta}{d t}=-D \nu \lambda^{2} \beta Z^{q-1}+k \lambda
$$

The solution for these two nonlinear coupled equations is

$$
Z(t)=Z_{0}\left[1+\frac{1}{k}\left(D \nu \lambda \beta_{0} Z_{0}^{q-1}-k\right)\left(1-e^{-k[\lambda+d(1-q)] t}\right)\right]^{d /[\lambda+d(1-q)]}
$$

and

$$
\beta(t)=\beta_{0}\left[1+\frac{1}{k}\left(D \nu \lambda \beta_{0} Z_{0}^{q-1}-k\right)\left(1-e^{-k[\lambda+d(1-q)] t}\right)\right]^{-\lambda /[\lambda+d(1-q)]},
$$

whose initial conditions are $Z(0)=Z_{0}$ and $\beta(0)=\beta_{0}$.

Since $\lambda+d(1-q)>0$, for positive $k$, we have a stationary solution and $\left\langle r^{2}\right\rangle$ goes to a constant value. When $k<0$, there is no stationary state and the $\left\langle r^{2}\right\rangle$ increases exponentially for large $t$. Our solution with $\theta=0$ corresponds to one obtained by Tsallis and Bukman [9] for the one-dimensional case when $\langle x\rangle=0$. We also remark that O'Shaugnessy-Procaccia equation, Eq. (5), when an external force is incorporated, is a special case of our results if we take $\nu=1$.

Before concluding this section, we note that in the presence of the source term, $-\alpha(t) \rho$, a similar procedure as presented in Sec. III can be employed, leading to Eq. (38) with $D$ replaced by $\hat{D}(t)$. Thus, Eqs. (39) and (40) must be solved with $\hat{D}(t)$ instead $D$ to obtain the generalized gaussian solution.

\section{STATIONARY SOLUTIONS FOR THE NONLINEAR FOKKER-PLANCK EQUATION}

We can extend our study about Fokker-Planck-like equation by investigating stationary solutions. The usual Fokker-Planck equation has a stationary solution that corresponds to the canonical Boltzmann-Gibbs distribution, $P \propto e^{-\beta V}$. On the other hand, the corresponding stationary solution of the nonlinear equation (2) is [16] 


$$
\rho(\mathbf{r})=\rho_{0}[1-(1-q) \beta V(\mathbf{r})]^{1 /(1-q)}
$$

if $1-(1-q) \beta V(\mathbf{r}) \geq 0$, and $\rho(\mathbf{r})=0$ if $1-(1-q) \beta V(\mathbf{r})<$ 0 . Here $\beta=\mu \rho_{0}^{q-1} /[(2-q) D], \rho_{0}$ is a positive integration constant, and $\mathbf{F}(\mathbf{r})=-\nabla V(\mathbf{r})$. Notice that $\beta>0$ when $V(\mathbf{r})$ is a confining potential.

When we consider the unified nonlinear equation

$$
\frac{\partial \rho(\mathbf{r}, t)}{\partial t}=\nabla \cdot\left\{K(\mathbf{r}) \nabla[\rho(\mathbf{r}, t)]^{\nu}\right\}-\nabla \cdot[\mu(\mathbf{r}) \mathbf{F}(\mathbf{r}) \rho(\mathbf{r}, t)],
$$

its stationary solution that generalizes Eq. (43) is

$$
\rho(\mathbf{r})=\rho_{0}\left[1-\left(\frac{1-q}{2-q}\right) \rho_{0}^{q-1} V_{e f f}(\mathbf{r})\right]^{1 /(1-q)}
$$

if $1-(1-q) \rho_{0}^{q-1} V_{e f f} /(2-q) \geq 0$, and $\rho(r)=0$ if $1-(1-q) \rho_{0}^{q-1} V_{\text {eff }} /(2-q)<0$, where we introduced the effective potencial $V_{\text {eff }}(\mathbf{r})=\int \frac{\mu(\mathbf{r})}{K(\mathbf{r})} \nabla V(\mathbf{r}) \cdot d \mathbf{r}$. Note that $[\mu(\mathbf{r}) / K(\mathbf{r})] \nabla V(\mathbf{r})$ must be written as a gradient so it is possible to obtain an effective potential in the $N$ dimensional case. In particular, for a 3-dimensional example, this implies that $\nabla[\mu(\mathbf{r}) / K(\mathbf{r})] \times \nabla V(\mathbf{r})=0$. This condition can be easily accomplished if we take $\mu=\mu(r)$, $K=K(r)$, and $V=V(r)$. Thus, Eq.(45) can be written as

$$
\rho(r)=\rho_{0}\left[1-\beta^{\prime}(1-q) V_{e f f}(r)\right]^{1 /(1-q)},
$$

with $\beta^{\prime}=\rho_{0}^{q-1} /(2-q)$ and $V_{\text {eff }}(r)=\int \frac{\mu(r)}{K(r)} \frac{d V(r)}{d r} d r$. Following the development presented in the previous sections, we stress that the stationary solution (46) remains true for an arbitrary non-integer dimension. For the onedimensional case, the solution (45) was obtained in Ref. [16.

\section{SUMMARY}

In this paper we have obtained exact spherically symmetric solutions for a nonlinear Fokker-Planck equation with radial-dependent diffusion coefficient, timedependent source, and spherical external force. We remark that the time-dependent solutions are generalizations of the gaussian ones.

Without both external force and source term, there is a competition between the fractal aspect and the nonlinear behavior (dictated by the parameter $\nu$ ), leading to superdiffusive, 'normal' and subdiffusive processes. On the other hand, in the presence of a source term, the mean square displacement is only affected in the nonlinear case, i.e., the source term modifies the superdiffusive, subdiffusive or 'normal' characters of the process when $\nu \neq 1$. In particular, this behavior is notorious when a power law time-dependent source is considered.

The diffusive process with a external force $F(r)=-k r$ leads to different behaviors to the time dependence of distribution function. For positive $k$, we have a stationary solution and the mean square displacement goes to a constant value. When $k<0$ there is no stationary state and the mean square displacement increases asymptotically in an exponential way.

In the case of the stationary solutions for our FokkerPlanck-like equation, we obtained a generalization of the canonical Boltzmann-Gibbs distribution. This generalized solution only exists if we are able to obtain an effective potential that includes the true potential, the drift and the diffusion coefficients.
[1] J. G. Berryman, J. Math. Phys. 18, 2108 (1977).

[2] M. F. Shlesinger, J. Klafter, and B. J. West, Physica A 140, 212 (1986); M. F. Shlesinger, B. J. West, and J. Klafter, Phys. Rev. Lett. 58, 1100 (1987).

[3] H. Spohn, J. Phys. (France) I 3, 69 (1993).

[4] J. Stephenson, Physica A 222, 234 (1995).

[5] Another representative approach to investigate anomalous diffusion is to consider integral equations (fractional derivatives). For instance, see J. Klafter, A. Blumen, and M. F. Shlesinger, Phys. Rev. A 35, 3081 (1987); R. Metzler, E. Barkai, and J. Klafter, Phys. Rev. Lett. 82, 3563 (1999); for a review see R. Metzler and J. Klafter, Phys. Rep. 339, 1 (2000).

[6] M. Muskat, The Flow of Homogeneous Fluids Through Porous Media (McGraw-Hill, New York, 1937).

[7] J. Buckmaster, J. Fluid Mech. 81, 735 (1977).

[8] J. M. Carlson, E. R. Grannan, C. Singh, and G. H. Swin- dle, Phys. Rev. E 48, 688 (1993).

[9] C. Tsallis and D. J. Bukman, Phys. Rev. E 54, R2197 (1996).

[10] A. R. Plastino and A. Plastino, Physica A 222, 347 (1995).

[11] D. A. Stariolo, Phys. Lett. A 185, 262 (1994); Phys. Rev. E 55, 4806 (1997);

[12] A. Compte and D. Jou, J. Phys. A 29, 4321 (1996).

[13] A. Compte, D. Jou, and Y. Katayama, J. Phys. A 30, 1023 (1997).

[14] S. Martinez, A. R. Plastino, and A. Plastino, Physica A 259, 183 (1998).

[15] L. Borland, Phys. Rev. E 57, 6634 (1998).

[16] L. Borland, F. Pennini, A. R. Plastino, and A. Plastino, Eur. Phys. J. B 12, 285 (1999).

[17] L. F. Richardson, Proc. R. Soc. London Ser. A 110, 709 (1926). 
[18] K. Takagoshi and H. Mori, Prog. Theor. Phys. 68, 439 (1982); H. G. E. Hentschel and I. Procaccia, Phys. Rev. A 27, 1266 (1983); H. G. E. Hentschel and I. Procaccia, Phys. Rev. A 29, 1461 (1984).

[19] B. O'Shaughnessy and I. Procaccia, Phys. Rev. Lett. 54, 455 (1985); B. O'Shaughnessy and I. Procaccia, Phys. Rev. A 32, 3073 (1985).

[20] L. C. Malacarne, R. S. Mendes, I. T. Pedron, and E. K. Lenzi, Phys. Rev. E 63, 030101(R) (2001).

[21] A. C. Scott, Rev. Mod. Phys. 47, 487 (1975).

[22] J. D. Murray, Mathematical Biology (Springer-Verlag,
Berlim, 1989).

[23] R. Aris, The Mathematical Theory od Diffusion and Reaction in Permeable Catalysts (Vol. I and II, Clarendon, Oxford, 1989).

[24] A. K. Myers-Beaghton and D. D. Vvedensky, Phys. Rev. B 42, 5544 (1990).

[25] G. Drazer, H. S. Wio, and C. Tsallis, Phys. Rev. E 61, 1417 (2000).

[26] G. E. Uhlenbeck and L. S. Ornstein, Phys. Rev. 36, 823 (1930). 